- helio and stellar seismology

- mm observations of the Sunyaev-Zeldovich effect, complementing balloon-borne submm observations

- site testing for image quality in the visible.

Such plans would preferably be carried out in an international base such as the one proposed by CARA at Dome A. The French plans for Antarctic infrastructure call for a base at Dome C, mostly optimized for geophysical observations.

\title{
ITALIAN PLANS FOR ASTROPHYSICAL OBSERVATIONS FROM ANTARCTICA
}

\author{
G Sironi, Universitá degli Studi, Milano, Italy \\ G Dall'Oglio,"La Sapienza" University, Roma, Italy
}

The characteristics which make Antarctica interesting for astrophysical research are: i) atmospheric transparency, ii) remoteness iii) geographical and geomagnetic position. Because of previous experience of observation from arctic sites in northern Europe and from isolated mountain sites, groups of Italian astrophysicists were immediately ready to go to Antarctica and bring their equipment to places not easily accessible, in a difficult environment when, about ten years, ago, the antarctic regions became more accessible.

With the support of the Italian Antarctic Program, two astrophysical projects are at present under way. 1) A permanent facility OASI (= Infrared and Submillimetric Antarctic Observatory) is in operation at Terra Nova Bay, the Italian base. Details about OASI were presented in a separate paper at this same conference (G Dall'Oglio 1991). 2) Observations of the Cosmic Background Radiation from the Amundsen Scott Base at South Pole are made by the University of Milano in collaboration with the Lawrence Berkeley Laboratory University of California/Berkeley. Both programs are expected to continue in 1991 and in the following years. In particular there are plans for regular observations at OASI and for an extension of the study of the Cosmic Background Radiation by adding to our radiometers, used to measure the CBR temperature, a polarimeter which will measure the degree of polarization (expected to be extremely small or null) of the CBR at $33 \mathrm{GHz}$.

Stimulated by the positive results so far obtained and by the expected improvements of the facilities available at Terra Nova Bay, in particular the transformation of the Base from only summer to a permanent station, new projects are now under consideration, namely: i) observations at $\mathrm{mm}$, sub- $\mathrm{mm}$ and in the infrared wavelength region (superheterodyne spectrometer for observation of molecular lines; source variability in the IR). ii) observations of the celestial background radiation at different wavelengths iii) studies of solar phenomena, in particular solar oscillations; in winter, monitoring of optical sources. iv) studies of the Cosmic Rays from ground level and at balloon altitudes (Cerenkov radiation in the earth's atmosphere and in the ice; opportunities offered by the reduced geomagnetic cut-off; search for rare components; long duration balloon flights around the polar cap). v) observations at the border between astrophysics, space physics and atmospheric physics, planned by collaborations between researchers of the three fields.

Amongst Italian astrophysicists who have been involved in observations in polar regions there is strong interest in projects for international facilities, completely dedicated to astronomy and astrophysics, supported and run by an International Institute. 